\title{
Breaker to Control Center Integration \& Automation: Protection, Control, Operation \& Optimization
}

\author{
Sakis Meliopoulos, George Cokkinides \\ School of Electrical and Computer Engineering \\ Georgia Institute of Technology
}

\author{
Evangelos Farantatos, Paul Myrda \\ EPRI
}

\begin{abstract}
Recent technological advances in protection, control and optimization are enabling a more automated power system. This paper proposes the use of these technologies towards an integrated and seamless infrastructure for protection, control and operation. This infrastructure is the basis for accommodating and providing robust solutions to new problems arising from the integration of renewables, namely more uncertainty and steeper ramp rates. At the lower level we propose a dynamic state estimation of a protection zone (EBP) for the purpose of providing protection for the zone. The estimation based protection (EBP) provides the real time dynamic model of the zone as well as the real time operating conditions. Since protection is ubiquitous, it can cover the full system. We assume that GPS synchronization of the EBP is available providing accurate time tags for the real time model and operating conditions. The real time model and operating conditions can extent from the "turbine to the toaster". We propose a methodology for automatically constructing the power system state locally and centrally at the control center with distributed controls as well as centralized controls depending on the application. For example, the centralized

system wide real time model is used to perform system optimization functions, and then send commands back through the same communication structure to specific power system components. Since protection is ubiquitous and the modern power system has several layers of communication infrastructure, the proposed approach is realizable with very small investment. The availability of the real time dynamic model and state locally and centrally enables the seamless integration of applications. Three applications are discussed in the paper: (a) settingless protection, (b) voltage/var control and (c) feeder load flexibility scheduling. The proposed approach and infrastructure can form the basis for the next generation of Energy Management Systems.
\end{abstract}

\section{Introduction}

The changing face of the electric power system due to new power apparatus and the proliferation of customer owned resources and smart devices calls for new approaches for protection, control and operation of the emerging electric power system. The promise of the smart grid is to provide technology to interconnect all these new resources for the common goal of operating the system efficiently and reliably and reward the owners of the various new and distributed resources. There are some new hard operating and protection constraints and realities. The grid is moving towards a system with many more power electronic devices that limit fault current and this trend compromises the existing protection functions that depend on substantial separation between fault currents and normal load currents. At the same time the emerging system requires better protection, more integration and more automation.

There is a need to rethink and update the overall approach for protection, control operation and optimization of the system. The objective of this paper is to propose an infrastructure and modeling standards for power systems that will enable the integration of seamless applications for the proper and timely protection, control, operation, and optimization of the power system. Each application may have specific time frame requirements; the proposed system addresses the required response times of these applications.

The proposed infrastructure consists of data acquisition systems that autonomously organize to perform autonomous state estimation using new modeling standards for the various components of the system and validate the operating state and real time models of the system. Since almost all control, operation and optimization functions are model based, the approach provides the required real time model throughout the geographical extent of the system and at time scales required by the various applications. The new modeling standards enable seamless development of the various applications for protection, control, operation and optimization. We describe the proposed system and provide example applications.

The paper is intended to generate discussion for the development of the next generation Energy Management 
System that will realize the objectives of the smart grid. It should be noted that the subject is vast and not all details can be covered in one technical paper. Two of the basic technologies on which the proposed approached is based, namely setting-less protection and distributed state estimation have been developed to a near commercial level and additional details can be found in the literature.

\section{Proposed Approach}

The overall proposed structure at the substation level is shown in Fig. 1. Later on in the section "System Wide Model Synthesis" we describe the approach at the control center level. The system starts from the relays that monitor power apparatus (a protection zone) and performs dynamic state estimation at the apparatus level. The dynamic state estimation is performed a few thousand times per second depending on the sampling period of the data acquisition systems. For example, if the relay samples 4800 times per second (an IEC standard), the dynamic state estimation is executed 2400 times per second (it uses two successive sampled data, see dynamic state estimation). This process is described in the section setting-less protection and it has been demonstrated with extensive numerical experiments and in the laboratory.

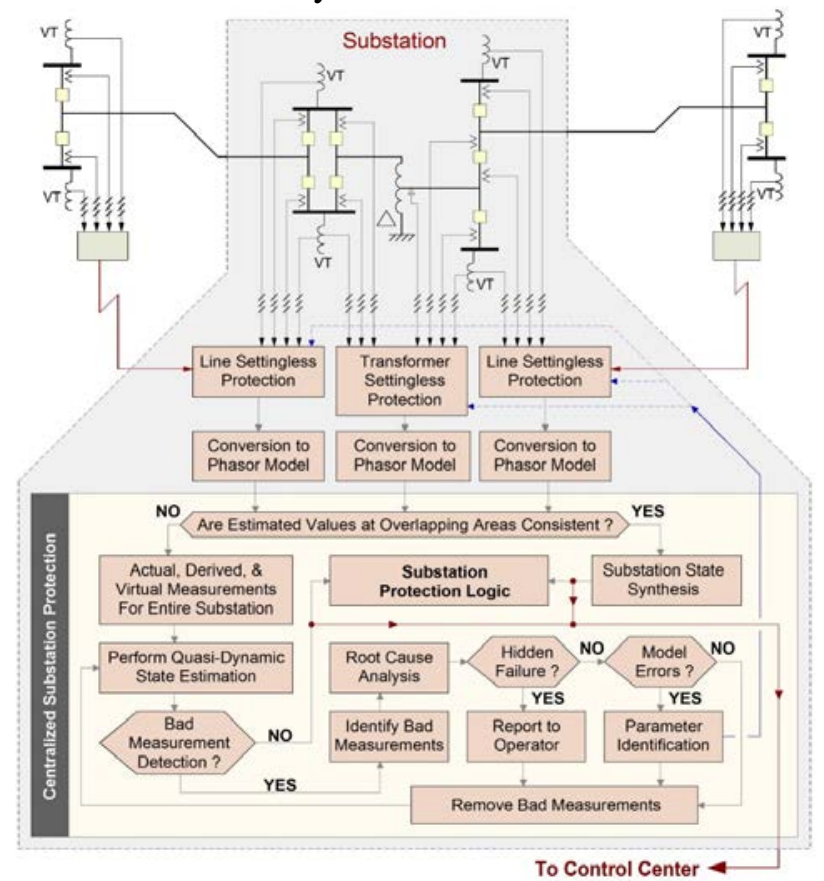

Figure 1: Illustration of Overall Approach at the Substation Level
The basic idea has been inspired from the differential protection function and can be considered as an extension and generalization of differential protection as it is illustrated in Figure 2. In differential protection the electric currents at all terminals of a protection zone are measured and their weighted sum must be equal to zero (generalized Kirchoff's current law). As long as the sum is zero or near zero no action is taking. In DSE based protection, all existing measurements in the protection zone are utilized. Specifically, currents and voltages at the terminals of the protection zone, as well as voltages, currents inside the protection zone (as in capacitor protection) or speed and torque in case of rotating machinery or other internal measurements including thermal measurements. Then, the dynamic model of the device (physical laws such as KCL, KVL, motion laws, thermodynamic laws, etc.) is used to provide the inter-relationship of all measured quantities. When there is no fault within the protection zone, the measurements should satisfy the dynamic model of the protection zone.

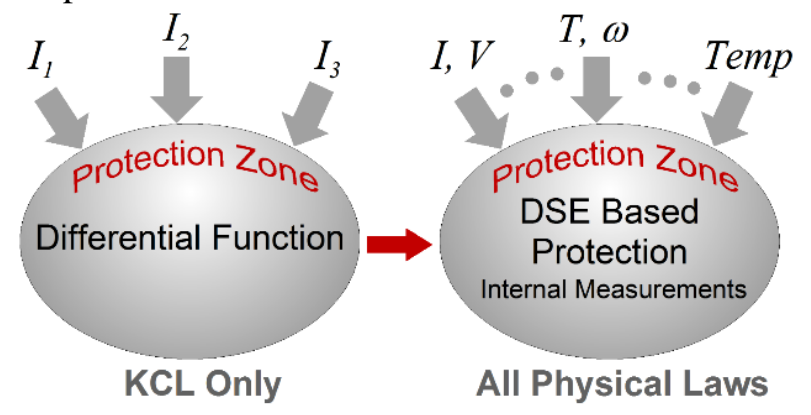

Figure 2: The DSE Based Protection Approach

A systematic way to verify that the measurements satisfy the mathematical model is a dynamic state estimation procedure. The resulting method is a Dynamic State Estimation Based Protective relay (EBP relay). When an internal fault occurs, even high impedance faults or faults along a coil, etc., the dynamic state estimation reliably detects the abnormality and a trip signal is issued with user selected controls, such as delay or reset. Three distinct dynamic state estimation algorithms (Extended Kalman Filter, Constraint Optimization and Unconstraint Optimization) have been developed and tested. Each algorithm requires the mathematical model of the protection zone, 
including instrumentation and the measurements. This basic approach has been extensively tested in the laboratory for several protection zones and presented in technical papers. It was coined settingless protection because of the simplified settings and it is void of coordination issues with other relays, the same way as differential protection does not require coordination.

The DSE based protective relay provides the following information: (a) the real time model of the protection zone and (b) the estimated voltage and current waveforms at each point of the protection zone. This information is used autonomously by upstream applications.

Real Time Model: It is provided in a standard syntax that we refer to as the SCAQCF (State and Control Algebraic Quadratic Companion Form (SCAQCF). This is a standard that we have developed to meet the requirements of the proposed automation. This standard provides the following information for the power apparatus (protection zone): ((1) connectivity, (2) device model, (3) measurements/data, (4) controls and (5) operating limits). The mathematical representation of the SCAQCF is:

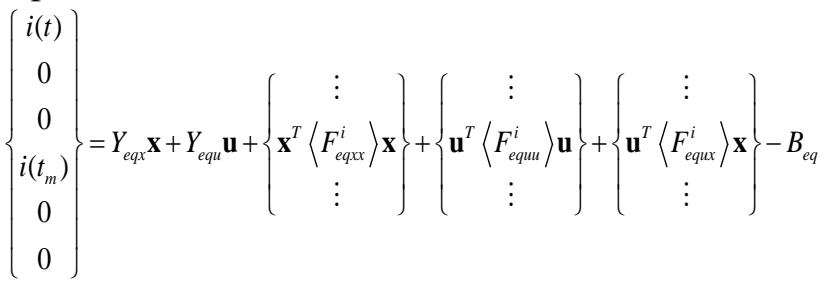

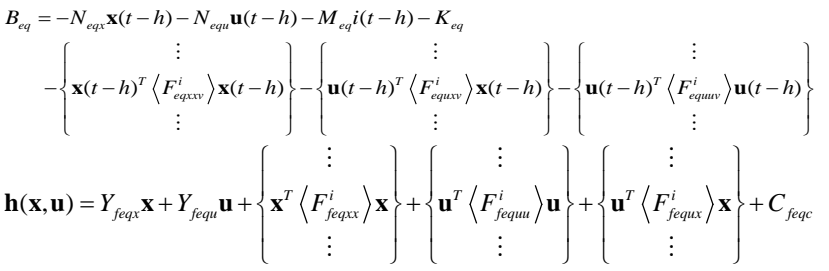

Connectivity: terminal node names: N1, N2, ...

subject to: $\quad \mathbf{h}_{\min } \leq \mathbf{h}(\mathbf{x}, \mathbf{u}) \leq \mathbf{h}_{\max }$

$$
\mathbf{u}_{\min } \leq \mathbf{u} \leq \mathbf{u}_{\max }, \quad \mathbf{x}_{\min } \leq \mathbf{x} \leq \mathbf{x}_{\max }
$$

where:

$\mathrm{i}(\mathrm{t})$ : the through variables of the device model.

$X$ : external and internal state variables of the device model,

$u$ : the control variables of the device model.

$B_{e q}$ : past history of device model.

The matrices shown are of appropriate dimensions and constant.
The main advantage of the SCAQCF model is that this formulation enables a generalized and abstract model of a fixed syntax for any component of the power system, which is independent of the type of the device and is suitable for implementation of object-oriented algorithms for any application. This generalization enables standardization for utilizing and exchanging the model of a device for other advanced smart grid applications in the proposed EMS which will be discussed in the following sections. Any new resource of component added to the system will be automatically accounted in the advanced application as long as its model is presented in the SCAQCF syntax.

States and Other Quantities: The sampled values of voltages and currents are converted into phasors and transmitted to applications and upstream systems such as the control center. A simplified example conversion of time domain model to phasor domain model is shown in Figure 3.

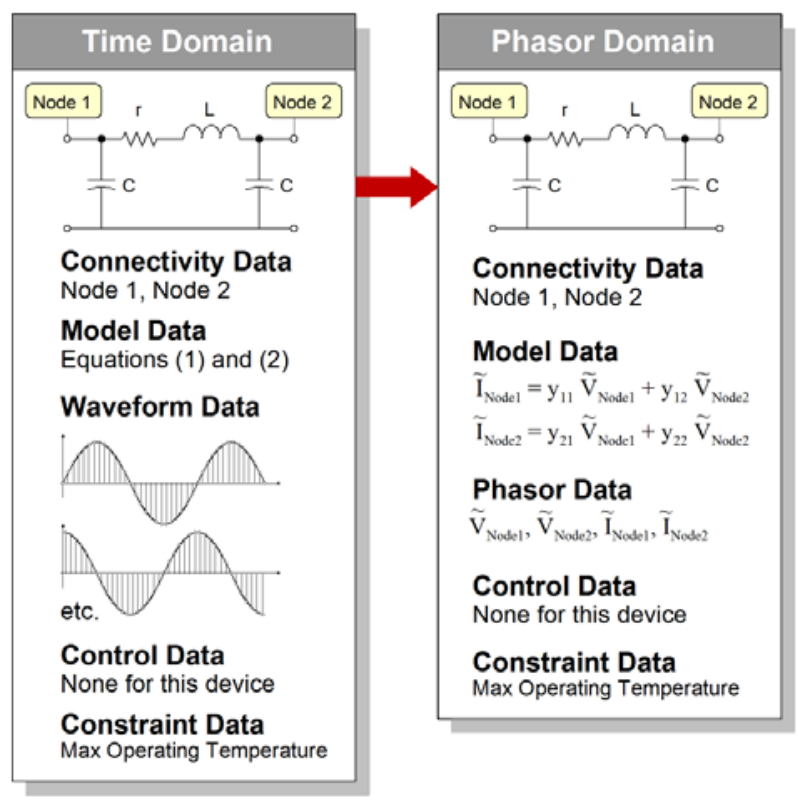

Figure 3: Simplified Example of Time Domain Model into Phasor Domain Model

Subsequently, this information is used to synthesize the substation state as shown in Fig. 1. Note that the substation state is updated once per cycle. Finally, the substation state is transmitted to the control center where the system state is synthesized, see Figure 4. Note that the synthesis of the substation state as well as the synthesis of the system state at the control center, does not require additional computations since the component models are all in 
UTC time (due to the GPS synchronized measurements) and therefore they can be simply merged to provide the system wide model.

Modeling accuracy and fidelity is fundamental for the DSE based protection. For many power system components, high fidelity models exist. For newer components such as inverter interfaced power components, the modeling accuracy may not be as high. In both cases the state estimation process can be utilized to fine tune the models and/or determine the parameters of the model with greater accuracy. These procedures have been demonstrated in [12]. The basic approach is to expand the dynamic state estimator to include some key model parameters as states to be estimated. Therefore, the overall approach can also provide better models with field validated parameters.

Since protection is ubiquitous, it makes economic sense to use relays as the gate keepers of the model of the device/protection zone they are protecting. By virtue of the DSE based protection, they also provide the capability of perpetually validating the model. The most important advantage is that the approach creates a depository of a high fidelity component model which in turn can provide the model for any possible application, from EMTP type studies to the simpler models required by control center applications.

\section{Substation Level State Estimation}

The results of the relay dynamic state estimation are in the form of estimated sample values of voltages and currents and other relevant quantities. We have described the procedure for converting the estimated sample values over a period of one cycle into phasor quantities. The algorithm which is shown symbolically in Figure 3 uses the "Standard PMU" [20]. The phasor model is expressed in terms of five sets of data: ((1) connectivity, (2) device model, (3) measurements, (4) controls, and (5) operating limits) that are time stamped. The syntax of the model is similar to the time domain model:

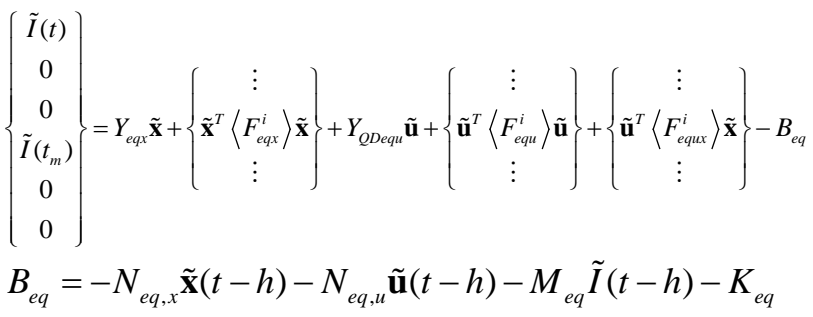

$\mathbf{h}(\tilde{\mathbf{x}}, \tilde{\mathbf{u}})=Y_{\text {feqx }} \tilde{\mathbf{x}}+Y_{\text {fequ }} \tilde{\mathbf{u}}+\left\{\begin{array}{c}\vdots \\ \left.\tilde{\mathbf{x}}^{T}\left\langle F_{\text {fequ }}^{i}\right) \tilde{\mathbf{x}}\right\} \\ \vdots\end{array}\right\}+\left\{\begin{array}{c}\vdots \\ \left.\tilde{\mathbf{u}}^{T}\left\langle F_{\text {fequ }}^{i}\right\rangle \tilde{\mathbf{u}}\right\} \\ \vdots\end{array}\right\}+\left\{\begin{array}{c}\vdots \\ \left.\tilde{\mathbf{u}}^{T}\left\langle F_{\text {fequx }}^{i}\right\rangle \tilde{\mathbf{x}}\right\} \\ \vdots\end{array}\right\}+C_{\text {feq }}$

Connectivity: TerminalNodeName

$$
\begin{array}{ll} 
& \mathbf{h}_{\min } \leq \mathbf{h}(\mathbf{x}, \mathbf{u}) \leq \mathbf{h}_{\max } \\
& \mathbf{u}_{\text {min }} \leq \mathbf{u} \leq \mathbf{u}_{\max }, \quad \mathbf{x}_{\min } \leq \mathbf{x} \leq \mathbf{x}_{\max }
\end{array}
$$

Subsequently, this information is used to synthesize the substation state estimate. This process is quite simple: the state estimates of each protection zone are aligned by the time stamp. The zone models of a specific time stamp are collected to form the substation state estimate. In our work we use a time interval of one cycle and therefore the substation state synthesis is updated once per cycle (in reality it is updated once per (1/f) seconds where $f$ is the nominal system frequency). Finally, the substation state is transmitted to the control center where the system state is synthesized. Note that the synthesis of the substation state does not require major additional computations since the component models are all in UTC time (due to the GPS synchronized measurements) and therefore they can be simply merged to provide the substation model.

\section{System Wide Model Synthesis at CC}

The substation state estimate (in frequency domain) is transmitted to the control center. At the control center the substation state estimates of exactly same time tag are put together to generate the system wide system estimate. The process is illustrated in Figure 4. Note that the figure shows one example of substation state estimator (substation 4) and the flow of data from all substations to the control center. The figure also shows the resulting system wide system visualization.

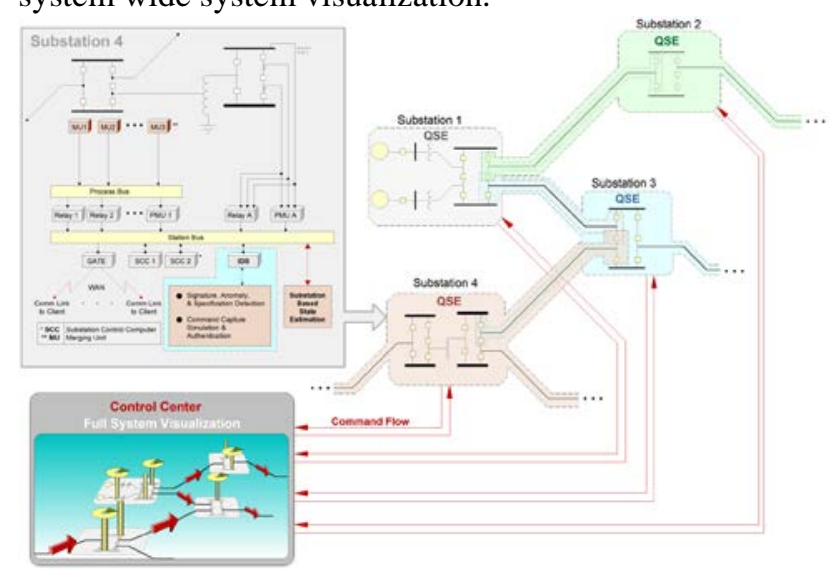

Figure 4. Flow of Data and System Wide Model/Estimate Synthesis

It is important to note that this approach does not require to perform a centralized state estimator at the control 
center assuming that (a) all substation state estimators are valid and (b) the time tag of each substation state estimator are accurate to GPS precision. It is also important to note that an independent verification is always performed as follows: the voltage at each end of a transmission line (three-phase voltages) are estimated from two independent estimators since the line connects two substations. These estimates must be within the accuracy of the metering for these voltages, otherwise a diagnostic is generated to investigate the discrepancy. Another option is to use the estimated substation quantities to run a centralized state estimator. Alternatively, the substation SE (running at the control center) can recreate all the measurements in the substation, thus maintaining the same redundancy as legacy centralized SE. It can be proven that this alternative does not provide additional information.

An important property of the distributed SE is that it is scalable to any size system since the computations are distributed to each substation and at the control center only the synthesis takes place. There are no major computations at the control center for this part of the system. This makes it possible to run a system wide state estimator at speeds of 60 times per second.

The substation SE sends to the EMS only its real time operating condition which comprises a very small number of data (the state of the substation), thus minimizing communications. If connectivity changes occur, then connectivity data are transmitted by exception. If model changes occur, the new mathematical model is transmitted by exception. The end result is that while the data collected at the substation are hundreds of thousands of data points per second, the frequency domain state (phasors) are only a few tens of data points per cycle transmitted to the EMS.

\section{Applications}

The proposed infrastructure and standards for representing the power system model provide in an autonomous manner a distributed system for seamless applications. These applications can range from local control problems, protection, monitoring the health and validity of data acquisition system and detecting hidden failures, to centralized system wide optimization, voltage/VAr control, scheduling, interchange evaluation and others. It is also important to note that the proposed infrastructure and modeling standards enable the applications to execute at the proper time frames, for example protection is executed at the sampled values rates, while voltage/VAr control at lower rates. It is impossible to discuss all the possible applications that can be seamlessly integrated in this framework. In previous publications we discussed the seamless integration of: (a) system protection, (b) stability monitoring, and (c) voltage/VAr control and optimization. In this paper we will discuss as an example the seamless integration of (a) centralized substation protection and (b) multi-step feeder optimization to take advantage of customer flexibility in the operation of the system.

\section{Application 1: Substation Centralized Protection and Detection of Hidden Failures}

The substation centralized protection and detection of hidden failures is shown in Figure 1. The system monitors the setting-less protection functions of all relays in a substation, collects all the data from all relays and using the redundancy in data detects and identifies hidden failures, if any. It supplements the setting-less protection to form a comprehensive system that is self-immunized against any type of failure, for example it can detect hidden failures such as blown fuses in the instrumentation channel and other usual hidden failures. A list of hidden failures is provided in Table 1 . In case of these failures, some of the collected data will be faulty. The dynamic state estimator, upon detection of these faulty data, it will replace the faulty data with estimated and validated data. The architecture of the system as shown in Figure 1. The first level is the setting-less relays which protect individual zones. The second layer is the substation level centralized system that monitors the operation of all setting-less relays in the substation (CSP). The CSP obtains the phasor quantities from setting-less relays and performs a dynamic state estimation for the entire substation as shown in Figure 1. The computed phasors are transmitted to the CSP through the station bus. This data represents redundant measurements relative to the state of the substation, typical redundancies are 2000\%. This means that there are 20 times more measurements than the number of states describing the operation of the substation. Because of this redundancy, bad data can be detected by simple tests, such as the chi-square test. When bad data is detected, they are traced back to the source of the problem (root cause) by using the dynamic model of the substation including the instrumentation.

Upon detecting the hidden failures, the CSP determines whether the bad measurements can be replaced with corrected measurements by virtue of the redundancy. If this is possible, the corrected data are sent to the settingless protection so that the system can operate without being affected by the hidden failures. At the same time, it sends a diagnostic to the operator to correct the failure. This way the system provides self-healing while it alerts the operator of the problem. This capability should be compared to present practice: some relays have limited capability to identify some types of hidden failures; in this case relay functions are inhibited and thus compromising the protection of the system. 
Table 1: Example Common Types of Hidden Failures

\begin{tabular}{|c|c|}
\hline Hidden Failure Type & Key Characteristic \\
\hline CT saturation & Harmonic Distortion \\
\hline CT short circuit & Zero current measurement \\
\hline CT wrong polarity & $180^{\circ}$ phase shift \\
\hline CT wrong ratio input & Lower current measurement \\
\hline PT blown fuses & $\begin{array}{c}\text { Zero voltage measurement for Y-Y } \\
\text { PT connection. } 50 \% \text { voltage } \\
\text { reduction for } \Delta-\Delta \text { PT connection }\end{array}$ \\
\hline
\end{tabular}

It is important to emphasize that, the computational requirements of the CSP is within the capability of modern computers. The concept has been demonstrated at the Georgia Tech laboratory (PSCAL). Response times are less than 1.5 cycles $(0.025 \mathrm{sec})$ which makes it suitable for protection functions.

\section{Application 2: Multi-Step Feeder Optimization}

Distribution systems with active components, such as responsive load, distributed storage and renewables, supplemented with thermostatically controlled loads have the capability to support the transmission grid and provide part of the required capacity reserve. Including distribution system resources in transmission level multiperiod economic dispatch is challenging due to the large number of devices. By extending the proposed infrastructure to feeders and possibly to residences, commercial building and industries and using the proposed modeling standards, the process of optimizing the feeder with all the customer resources becomes a seamless application. Because these resources have effective storage capability, the optimization is performed over a period of time, the planning horizon. The resulting optimization problem is a multi-step optimal power flow, or the Flexible OPF as we have name it [18], [19]. The Flexible OPF optimally dispatches the distribution system's active \& reactive support to the grid, over a look-ahead horizon.

One of the fundamental challenges in formulating and solving the flexible OPF problem is the capability to model a wide range of power system device models, in addition to the standard models of generators, transmission lines, transformer and loads. Indeed, an effective flexible OPF framework must include models for a wide range of storage technologies, explicit models of responsive loads with customer convenience constraints, as well as new dynamic models for classical devices, such as ramp-limited generators and dynamic thermal line ratings. In the context of this work, this issue is addressed by introducing "object-oriented" device models that describe the dynamics, algebraic equations and constraints of each device. The device modeling is completely decoupled from the algorithms used to handle the device's connectivity in the network, as well as the algorithms for the synthesis and solution of the optimization problem. In the context of the proposed infrastructure, the device models will be part of the protective relay which will continuously will be matching the model to the streaming measurements, thus continuously validating the model. The model object will be passed to the Flexible OPF when the Flexible OPF is initiated. Note that this seamless operation will guarantee that the Flexible OPF will start from the present real time model of the system as well as the present operating condition. The process also guarantees that the model used to setup the Flexible OPF will be always validated.

The Flexible OPF is formulated as follows:

$$
\underset{\mathbf{X}, \mathbf{U}}{\operatorname{Minimize}} \sum_{k=1}^{K} c\left(\mathbf{X}_{k}, \mathbf{U}_{k}\right)
$$
subject to:

$$
\begin{array}{cc}
\mathbf{g}\left(\mathbf{X}_{k-1}, \mathbf{U}_{k-1}, \mathbf{X}_{k}, \mathbf{U}_{k} \mid \mathbf{P}_{k}\right)=0 & k=1,2, \ldots, K \\
\mathbf{h}\left(\mathbf{X}_{k-1}, \mathbf{U}_{k-1}, \mathbf{X}_{k}, \mathbf{U}_{k} \mid \mathbf{P}_{k}\right) \leq 0 & k=1,2, \ldots, K \\
\mathbf{U}_{\text {min }} \leq \mathbf{U}_{k} \leq \mathbf{U}_{\max } & k=1,2, \ldots, K \\
\mathbf{X}_{\min } \leq \mathbf{X}_{k} \leq \mathbf{X}_{\max } & k=1,2, \ldots, K \\
\mathbf{X}_{0}=\mathbf{X}_{\text {init }} & \\
\mathbf{U}_{0}=\mathbf{U}_{\text {init }} &
\end{array}
$$

Where the objective function is the sum of the costs of operating the system at each one of the intervals $k$, the equality constraints $g(. .$.$) are autonomously constructed$ from the SCAQCF models of the various components of the system and the constraints are simply assembled from the constraints of the SCAQCF model [18], [19]. It is important to note that these constraints included ramp limits for some generators, no inconvenience constraints for customers and many newer requirements.

The flexible OPF problem is a quadratically constrained quadratic program (QCQP). Note that $\mathbf{X}_{k}, \mathbf{U}_{k}$ and $\mathbf{P}_{k}$ are the consolidated system state, control and parameter vectors respectively. The parameter vector depends on the particular device models employed. The flexible OPF needs to be solved independently for each possible scenario in the look-ahead $K$-step horizon. Sample results are presented in Figure 5.

\section{Conclusions}

The basic concept and promise of the smart grid is to provide more automation and take advantage of the flexibility in the system to enable more secure and reliable operation as well as more economical operation. 
An important realization is that new trends of ever increasing presence of power electronics throughout the system is affecting fault current levels and it is compromising protection systems. To achieve a more secure, reliable and economic operation, it is important to remove human intervention or needs for human input as much as possible to avoid the possibility of human error as the operation of the system becomes more complex and the number of players is increasing. This paper proposes an infrastructure and a modeling standard that enables fully autonomous protection, control, operation and optimization of the system by seamless and autonomous integration of applications. We have presented two examples of seamless applications, one in the area of system protection and another in the area of optimizing the flexibility of the system. Practically, any of the usual applications that are used for the system can be seamlessly integrated into the proposed framework.
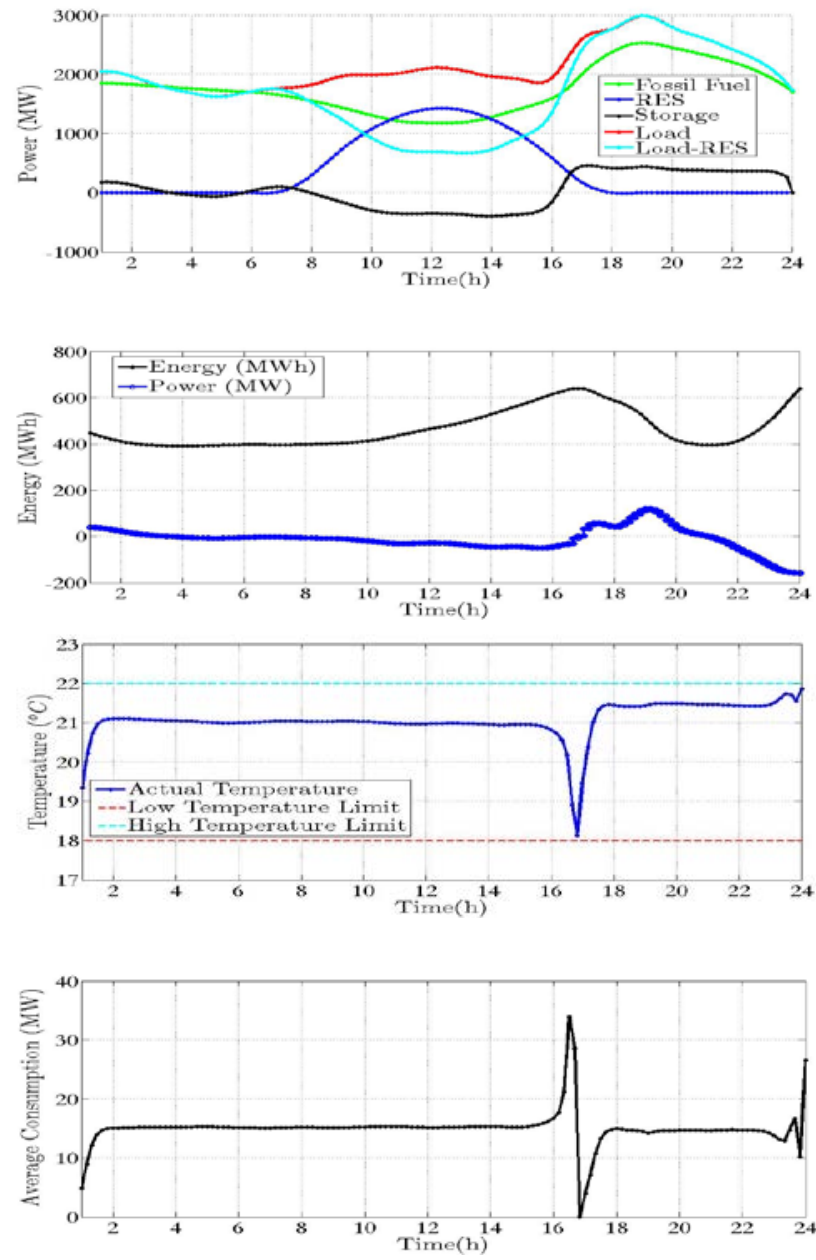

Figure 5: Example Results of Feeder Flexibility Optimization (Flexible OPF) and Impact on System Resources

(First Figure: Primary Resources Utilization and Ramp Rates, Second Figure: Charge/Discharge Schedule of PHEVs, Third Figure:

Temperature of Thermostatically Controlled Loads, and Forth Figure: Consumption of Thermostatically Controlled Loads)
The infrastructure requires that each protective relay be loaded with the dynamic model of the physical system that protects. While this may appear to be a complex task, in reality it is not. It is not difficult to include the motor model in motor relays, the transformer model in transformer relays or the air-conditioning model in A/C protection systems. Each relay can also fine tune the parameters of the model and provide the validated models to concentrators that can form the aggregate models depending what the end use or application is. This also solves the problem of modeling the total load of the system, an area that is needed for many applications. The proposed infrastructure will provide detailed mathematical models of loads and these models can be aggregated to load models for operations and planning purposes [19].

\section{References}

[1] H. L. Storey, "Implementing an Integrated Centralized ModelBased Distribution Management System,” in Proc. 2011 IEEE PES General Meeting, Detroit, MI, July 2011.

[2] A. Ipakchi, "Demand side and distributed resource management A transactive solution,” in Proc. 2011 IEEE PES General Meeting, Detroit, MI, July 2011.

[3] A. P. Meliopoulos, G. J. Cokkinides, Z. Tan, S. Choi, Y. Lee and P. Myrda, "Setting-less Protection: Feasibility Study", in Proc. 2013 46th Hawaii International Conference on System Sciences (HICSS), pp. 2345-2353, Maui, HI, Jan. 2013.

[4] A. P. Meliopoulos, G. J. Cokkinides, S. Choi, E. Farantatos, R. Huang and Y. Lee, "Symbolic integration and autonomous state estimation: Building blocks for an intelligent power grid", in Proc. 16th International Conference on Intelligent System Application to Power Systems (ISAP), Greece, Sept. 2011.

[5] A. P. Meliopoulos, G. J. Cokkinides, and G. K. Stefopoulos, "Symbolic integration of dynamical systems by collocation methods," in Proc. 2005 IEEE-PES General Meeting, pp. 817-822.

[6] A. P Meliopoulos, G. Cokkinides, R. Huang, E. Farantatos, S. Choi, Y. Lee, and X. Yu, "Smart Grid Technologies for Autonomous Operation and Control”, IEEE Transactions on Smart Grid, Vol. 2, Issue 1, 2011.

[7] S. Choi, B. Kim, G. J. Cokkinides, A. P. Meliopoulos, "Feasibility Study: Autonomous State Estimation in Distribution Systems", IEEE Trans. on Power Systems, Vol. 2, Issue 1, 2011.

[8] R. Huang, E. Farantatos, G. Cokkinides, A. P. Meliopoulos, B. Fardanesh and G. Stefopoulos, "Object-Oriented 3-Phase Distributed Dynamic State Estimator,” in Proc. 2011 IEEE PES General Meeting, Detroit, MI, July 2011.

[9] A. P. S. Meliopoulos, G. J. Cokkinides, C. Hedrington, and T. L. Conrad, "The SuperCalibrator - A Fully Distributed State Estimator", in Proc. 2010 IEEE PES General Meeting, Minneapolis, MN, July, 2010.

[10] A. P Meliopoulos, G. Cokkinides, R. Huang, E. Farantatos, S. Choi and Y. Lee, "Wide Area Dynamic Monitoring and Stability August Controls", in Proc. 2010 Bulk Power System Dynamics and Control Symposium, Buzios, RJ, Brazil, August 2010.

[11] PSRC Working Group K15, "Centralized Substation Protection and Control", December 2015.

[12] Protection System Misoperation Task Force, "Misoperations Report,” NERC Planning Committee, 2014.

[13] Q. Qiu, "Risk assessment of power system catastrophic failures and hidden failure monitoring \& control system," PhD 
Dissertation, Virginia Polytechnic Institute and State University, 2003.

[14] PSERC Report 13-46, "Setting-less Protection".

[15] S. Meliopoulos, G. J. Cokkinides, S. Grijalva, R. Huang, E. Polymeneas, P. Myrda, E. Farantatos, and M. Gehrs, "Integration \& automation: From protection to advanced energy management systems," Proceedings of Bulk Power System Dynamics and Control-IX Optimization, Security and Control of the Emerging Power Grid (IREP), 2013 IREP Symposium, 2013, pp. 1-11.

[16] Sakis Meliopoulos, G. J. Cokkinides, R. Huang and P. Myrda, "Grid Modernization: Seamless Integration of Protection, Optimization and Control," 2014 47th Hawaii International Conference on System Sciences, Waikoloa, HI, 2014, pp. 24632474.

[17] S. Brahma, "Advancements in Centralized Protection and Control within a Substation," in IEEE Transactions on Power Delivery, Volume: PP, Issue: 99, pp.1-1.

[18] Sakis Meliopoulos, Evangelos Polymeneas and Renke Huang, "Flexible Resource Optimization to Mitigate Operational Problems from Variable Generation", Proceedings of the PowerTech Eindhoven 2015, Eindhoven, The Nederlands, June 29-July 2, 2015.

[19] Evangelos Polymeneas, A. P. Meliopoulos, "Aggregate Modeling of Distribution Systems for Multi-Period OPF," Proceedings of the PSSC 2016, Genoa, Italy, June 20-24, 2016.

[20] Anurag Srivastava, Sakis Meliopoulos and Pete Sauer, "Adaptive and Intelligent PMU for Smarter Applications", PSERC Publication 16-01, January 2016.

A. P. Sakis Meliopoulos (M '76, SM '83, F '93) was born in Katerini, Greece, in 1949. He received the M.E. and E.E. diploma from the National Technical University of Athens, Greece, in 1972; the M.S.E.E. and Ph.D. degrees from the Georgia Institute of Technology in 1974 and 1976, respectively. In 1971, he worked for Western Electric in Atlanta, Georgia. In 1976, he joined the Faculty of Electrical Engineering, Georgia Institute of Technology, where he is presently a Georgia Power Distinguished Professor. He is active in teaching and research in the general areas of modeling, analysis, and control of power systems. He has made significant contributions to power system grounding, harmonics, and reliability assessment of power systems. He is the author of the books, Power Systems Grounding and Transients, Marcel Dekker, June 1988, Lightning and Overvoltage Protection, Section 27, Standard Handbook for Electrical Engineers, McGraw Hill, 1993. He holds three patents and he has published over 220 technical papers. In 2005 he received the IEEE Richard Kaufman Award and in 2010 received the George Montefiori Award. Dr. Meliopoulos is the Chairman of the Georgia Tech Protective Relaying Conference, a Fellow of the IEEE and a member of Sigma Xi.

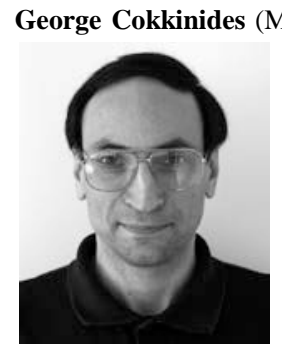

'85) was born in Athens, Greece, in 1955. He obtained the B.S., M.S., and Ph.D. degrees at the Georgia Institute of Technology in 1978, 1980, and 1985, respectively. From 1983 to 1985, he was a research engineer at the Georgia Tech Research Institute. Since 1985, he has been with the University of South Carolina where he is presently an Associate Professor of Electrical Engineering. His research interests include power system modeling and simulation, power electronics applications, power system harmonics, and measurement instrumentation. Dr. Cokkinides is a member of the IEEE/PES.
Evangelos Farantatos (St. M '06, M '13) was born in Athens, Greece in 1983. He received the Diploma in Electrical and
Computer Engineering from the National Technical University of Athens, Greece, in 2006 and the M.S. in E.C.E. and Ph.D. degrees from the Georgia Institute of Technology in 2009 and 2012 respectively. He is currently a Sr. Project Engineer/Scientist at EPRI. In summer 2009, he was an intern in Midwest ISO. His research interests include power systems state estimation, protection, stability, operations, control, renewables integration and smart grid technologies.

Paul Myrda is a Technical Executive with EPRI and manages the

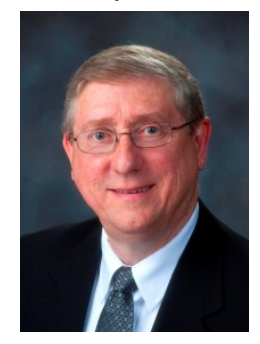
Information Communication Technology Transmission program and Grid Transformation project. Paul represents EPRI on Advisory Boards for the Power Systems Engineering and Research Council, Trustworthy Cyber Infrastructure for the Power Grid Center and North American Synchrophasor Initiative. He has an MBA from Kellogg; MSEE and BSEE from Illinois Institute of Technology, licensed professional engineer, and member of IEEE and CIGRE. 